\title{
Commentary: Clear as mud: Truncus arteriosus and major (check for updates adverse cardiac events
}

\author{
Tara Karamlou, MD, MSc
}

\author{
From the Division of Pediatric Cardiac Surgery and Heart Vascular Institute, Cleveland Clinic, Cleveland, Ohio. \\ Disclosures: Author has nothing to disclose with regard to commercial support. \\ Received for publication Jan 13, 2019; accepted for publication Jan 14, 2019; available ahead of print March 7 , \\ 2019. \\ Address for reprints: Tara Karamlou, MD, MSC, Division of Pediatric Cardiac Surgery, Cleveland Clinic, Heart \\ Vascular Institute, 9500 Euclid Ave, Cleveland, OH 44120 (E-mail: karamlt@ccf.org). \\ J Thorac Cardiovasc Surg 2019;157:2402-3 \\ $0022-5223 / \$ 36.00$ \\ Copyright (c) 2019 by The American Association for Thoracic Surgery \\ https://doi.org/10.1016/j.jtcvs.2019.01.053
}

The article in this issue of the Journal by Mastropietro and colleagues ${ }^{1}$ investigates in-hospital adverse outcomes and their determinants among patients with truncus arteriosus undergoing complete primary repair at 15 selected institutions from 2009 to 2016. Among the 216 patients included, the in-hospital mortality was $6.9 \%$, and the prevalence of major adverse cardiac events (MACE), defined as intraoperative or perioperative cardiopulmonary resuscitation, extracorporeal membrane oxygenation, or death, was $20 \%$. Mastropietro and colleagues ${ }^{1}$ found that implantation of a right ventricle-to-pulmonary artery conduit with an indexed diameter larger than $50 \mathrm{~mm} / \mathrm{m}^{2}$, duration of cardiopulmonary bypass (CPB) longer than 150 minutes, and discharge home from the nursery before diagnosis were risk factors for MACE.

It is well known that repair of truncus arteriosus (currently a STAT [The Society of Thoracic SurgeonsEuropean Association for Cardio-Thoracic Surgery] category 4 procedure) carries a significant risk of mortality, even in the contemporary era. Still, that one-fifth of babies will have MACE occur reminds all of us that considerable attention (in terms of quality improvement efforts, refinements in perioperative care, and improved diagnostic paradigms) are critically needed among this challenging population. It is therefore laudable that Mastropietro and colleagues ${ }^{1}$ have focused on this group, given the attention currently devoted to the single-ventricle population.

Despite the importance of the subject matter of this study and the excellent composition of the article, there are several inconsistencies and "oddities" that muddy the overall message. The first relates to the unsurprising finding that prolonged CPB was associated with worse outcomes. Similar to the 208 other articles within the last 5 years that have been published under the search terms "cardiopulmonary bypass time," "outcomes," and "congenital," the risk factor of CPB longer than 150 minutes is likely a surrogate for other predictors that remain hidden and therefore unaddressed. ${ }^{2}$ CPB time is a continuous predictor, and it will be chosen preferentially by most automated selection

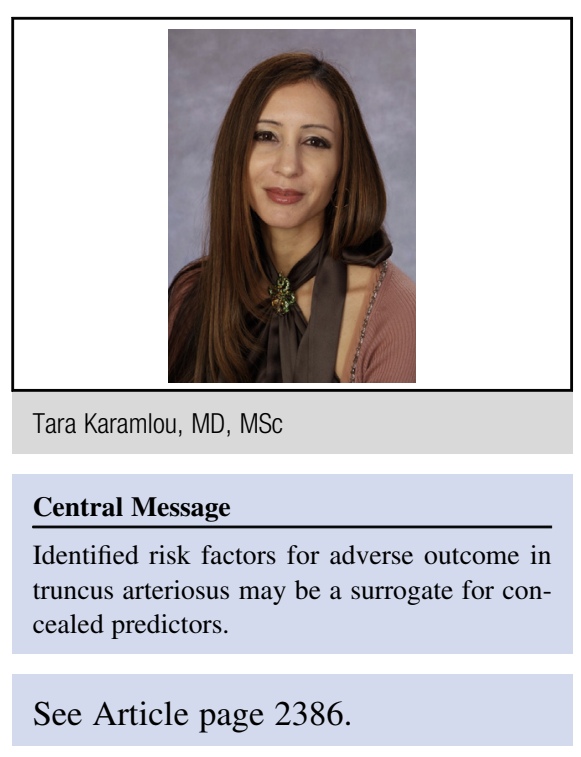

algorithms unless particular attention is directed at "finding the truth." Mastropietro and colleagues ${ }^{1}$ did attempt to parse out the collinear factors that could lead to the emergence of lengthy CPB as a risk factor, including lower target temperature, cooling and rewarming protocols, ultrafiltration, use of deep hypothermic circulatory arrest, or need to address concomitant defects. I remain concerned, however, that longer CPB time in this study is at best a distractor and at worst may obviate identification of modifiable management pearls. Review of the data within this study demonstrate that MACE occurred among the 3 patients in which truncal valve repair was attempted before eventual truncal valve replacement during a second CPB exposure. The CPB times among these neonates were 333, 336, and 356 minutes. Contrastingly, neonates who underwent truncal valve replacement a priori, without initial attempts at repair (CPB times of 217, 291, and 323 minutes) had a $0 \%$ prevalence of MACE. It may therefore be that avoiding a second CPB exposure could be accomplished with identification of those truncal valve characteristics that mitigated performance of a successful repair. In my mind, it is these types of data that could lead to modification of surgical practice and improved results.

It is understandably difficult to dissect out whether reductions in CPB times are feasible or even important, given the disparate literature on the subject and the variations in surgical technique used to repair most complex defects. One 
strategy to potentially determine whether prolonged $\mathrm{CPB}$ times are surrogates for complexity of a particular operation and not associated with a particular surgical practice or surgeon is to determine whether the prolonged CPB times longer than 150 minutes were outside the median CPB times for the participating surgeons. Outliers are more likely to be related predominantly to particular patient factors and therefore are much less likely to be ideal targets for performance improvement initiatives. Given the design of the study, which involved solicitation of centers by the investigative team, presumably Mastropietro and colleagues ${ }^{1}$ had the luxury of harvesting such data from participating centers rather than being relegated to reliance on registry data, which would hinder the ability to perform such analyses. Perhaps the follow-up from this dedicated group will provide some much-needed answers to these complex questions.

The second perplexing finding in this article was that "failure to diagnose truncus before discharge from the nursery" was associated with MACE but not associated with preoperative shock. Mastropietro and colleagues ${ }^{1}$ hypothesize that ensuing "advanced compensated heart failure" may have been the predominant clinical picture. Given that the pathophysiology of truncus arteriosus involves compromised systemic perfusion, owing to preferential flow to the pulmonary artery bed (especially in the presence of truncal valve insufficiency), however, this contention seems unlikely. Certainly, the Society of Thoracic Surgeons' definition of preoperative shock $(\mathrm{pH}<7.2$ and lactate level $>4 \mathrm{mmol} / \mathrm{L}$ ) may be too lenient to detect early or more subtle shades of preoperative shock; however, leveraging other diagnostic modalities, such as near-infrared spectroscopic monitoring, or providing echocardiographic evidence of systemic ventricular function or left ventricular dilation could lend credence to this perspective. Ultimately, the most important point that the study by Mastropietro and colleagues ${ }^{1}$ underscores is to reveal the fallacy that babies (whether apparently "stable" or not) can be discharged and managed at home before definitive repair. The $71 \%$ readmission rate for cardiorespiratory failure among the 17 patients managed with this strategy is testament to this fact. The clinical imperative that neonatal repair of truncus arteriosus be the standard to which all programs are held in the contemporary era should be emphasized.

I found it interesting (and, frankly, enviable) that the prenatal detection rate among the babies in this study was $63 \%$. This is nearly double the published prenatal detection rates in many areas of the Unites States, such as California or Arizona, where it is has remained around $31 \%$ to $35 \%$ despite comprehensive, multidisciplinary, and thoughtful efforts. ${ }^{3}$ It is well known that study participants likely represent a higher sociodemographic strata with increased access to prenatal care; this fact notwithstanding, it would have been very instructive to understand the perinatal care paradigms and infrastructure at the participating centers that may have contributed to their comparatively high prenatal detection rates. Moreover, given that failure to diagnose before discharge was a risk factor for MACE (but not for mortality), it is likely that neonates without prenatal diagnosis may represent a much larger population across the United States than suggested by the study of Mastropietro and colleagues.

The association of larger indexed conduit size with MACE, with an inflection point at $50 \mathrm{~mm} / \mathrm{m} 2$, is mentioned many times throughout the paper. Indexing a conduit to body surface area, as opposed to indexing to patient weight or normalization with $z$ scores, is uncommon in the neonatal population. Mastropietro and colleagues ${ }^{1}$ provide a reasonable rationale for electing to use body surface area, and this is indeed one of the novel aspects of their study. The use of conduit size as a linear predictor, however, is an incomplete representation given that an ideal conduit is neither too small nor too large. In other words, constraining the variable to a linear relationship with the outcome fails to capture the nonlinear dynamics of all biologic systems. ${ }^{4}$ Mastropietro and colleagues. ${ }^{1}$ should have explored natural cubic splines or other polynomial transformations that might have clarified the "optimum physiologic window" of conduit size. Indeed, this exact concept was harnessed in the initial Congenital Heart Surgeons' Society article that prospectively investigated determinants of conduit dysfunction in 241 infants younger than 2 years at initial conduit implantation. ${ }^{5}$ Karamlou and colleagues ${ }^{5}$ used polynomial transformations of initial conduit $z$ score and found that conduit failure was accelerated when the conduit $z$ score was less than +1 or greater than +3 . It would be interesting to use these same methods to validate the novel use by Mastropietro and colleagues ${ }^{1}$ of body surface area to index conduit size in neonates. In this way, we can build on promising formative work...iterative improvement and refinement to expedite forward progress. We do not need to reinvent the wheel, just realign it.

\section{References}

1. Mastropietro CW, Amula V, Sassalos P, Buckley JR, Smerling AJ, Iliopoulos I, et al. Characteristics and operative outcomes for children undergoing repair of truncus arteriosus: a contemporary multicenter analysis. J Thorac Cardiovasc Surg. 2019;157:2386-98.e4.

2. US National Library of Medicine. PubMed MeSH Browser search terms cardiopulmonary bypass time, outcome, congenital. Available at: https://www.ncbi. nlm.nih.gov/pubmed/?term $=$ cardiopulmonary + bypass + time $\% 2 \mathrm{C}+$ outcome $\%$ 2C + congenital. Accessed January 5, 2019.

3. Quartermain MD, Pasquali SK, Hill KD, Goldberg DJ, Huhta JC, Jacobs JP, et al Variation in prenatal diagnosis of congenital heart disease in infants. Pediatrics. 2015; $136:$ e378-85.

4. Janson NB. Non-linear dynamics of biologic systems. Contemp Phys. 2012;53: 137-68.

5. Karamlou T, Blackstone EH, Hawkins JA, Jacobs JP, Kanter KR, Brown JW, et al; Pulmonary Conduit Working Group for the members of the Congenital Heart Surgeons Society. Can pulmonary conduit dysfunction and failure be reduced in infants less than age 2 years at initial implantation? J Thorac Cardiovasc Surg. 2006;132:829-38.

6. D'Angelo AJ. The Principle of Change. Available at: https://georgecouros.ca/ blog/archives/tag/anthony-j-dangelo. Accessed January 2, 2019. 